\title{
The Degenerating Substantia Nigra as a Susceptible Region for Gene Transfer-Mediated Inflammation
}

\author{
Valeria Roca, Juan Cruz Casabona, Pablo Radice, Verónica Murta, \\ and Fernando Juan Pitossi
}

Leloir Institute, IIBBA-CONICET, Avenida Patricias Argentinas 435, 1405 Buenos Aires, Argentina

Correspondence should be addressed to Fernando Juan Pitossi, fpitossi@leloir.org.ar

Received 2 December 2010; Accepted 29 March 2011

Academic Editor: Gilles J. Guillemin

Copyright (c) 2011 Valeria Roca et al. This is an open access article distributed under the Creative Commons Attribution License, which permits unrestricted use, distribution, and reproduction in any medium, provided the original work is properly cited.

\begin{abstract}
Parkinson's disease (PD) is characterized by the progressive degeneration of neurons in the substantia nigra pars compacta (SN). The naïve SN is highly susceptible to inflammation. In addition, microglial activation in the degenerating SN displays distinct characteristics that increase the reactivity of the region towards inflammatory stimuli. On the other hand, gene therapy for PD has recently move forward into clinical settings, with PD being the neurodegenerative disorder with the highest number of Phase I/II gene therapy clinical trials approved and completed. These clinical trials are not targeting the SN, but this region is a certain candidate for future gene therapy interventions. Here, the unique immune-related properties of the degenerating SN in the context of a putative gene therapy intervention are reviewed. Several variables affecting the host response to gene delivery such as vector type and dosage, age and stage of disease of patients, and method of gene delivery and transgene used are discussed. Finally, approaches to diminish the risk of immune-mediated toxicity by gene transfer in the SN are presented.
\end{abstract}

\section{Introduction}

Parkinson's disease (PD) is a neurodegenerative disorder characterised by the progressive loss of dopaminergic neurons in the substantia nigra pars compacta (SN) (reviewed in [1]). The aetiology of the most common forms of PD remains unknown. Current therapeutic treatments comprise pharmacological strategies to compensate for dopamine deficiency or surgical interventions that reduce the hyperactivity of specific regions within the basal ganglia (reviewed in [2]). However, dopamine replacement can lead to undesired sideeffects 5-10 years after the beginning of treatment [3]. As no treatment is available that can prevent disease progression, the search for new therapeutic interventions is intense. In particular, gene therapy approaches have successfully reached the clinical trial stage in a number of cases [4]. Approved gene therapy clinical trials are based on restoring the activity of the basal ganglia by providing growth factors, inhibiting hyperactive regions, or enhancing dopamine synthesis [4].

Viral gene delivery seems to be the method of choice for gene therapy for PD due to its high efficiency for gene transduction. A drawback to the delivery of genes via viral vectors comes by introducing an antigenic load into the brain. These antigens will invariably elicit a transient innate immune response [5]. The nature and functional (toxic or protective) consequences of this response will vary depending on a number of variables but of utmost importance is the region of gene transfer, the viral dose used, and the state of microglial activation in that region [5]. Importantly, the $\mathrm{SN}$ is highly susceptible to the toxic effects of inflammation $[6,7]$. In addition, microglial activation during neurodegeneration in this region possesses particular features that could exacerbate disease progression if a proinflammatory stimulus hits the SN [8].

This paper will focus on the properties of microglial activation in the degenerating SN in PD. In addition, the immune reaction after gene delivery by adenoviral, adenoassociated, and lentiviral vectors in the CNS will be discussed. Finally, a list of risk factors and parameters that could be considered when assessing the possible influence of gene transfer to the $\mathrm{SN}$ is presented as well as alternative approaches to circumvent inflammation-mediated toxicity. 


\section{Inflammation in the Central Nervous System}

Inflammation in the Central Nervous System (CNS) has different features according to (i) the region in which it occurs, (ii) the stimulus, and (iii) the molecular and cellular milieu at the time of the response. For example, inflammation in the brain parenchyma is usually restricted to certain leukocyte populations, harder to initiate, and less widespread than inflammation when it occurs in the ventricles, meninges, and choroid plexus [9]. At these sites the characteristics are more reminiscent of a typical systemic inflammatory response. This difference is mainly due to the absence of dendritic cells, conventional lymphatics, the downregulation of major histocompatibility complex (MHC) molecules within the CNS parenchyma, and the presence of local immunosuppressive factors (reviewed in [4, 9-11]). In addition, the innate inflammatory response in the CNS parenchyma does not always lead to an activation of the adaptive arm of the immune system (reviewed in [12]).

By origin and function, microglial cells can be regarded as the resident macrophages of the brain and are the main innate immune cells in the CNS. Microglial activation is a highly dynamic process [13-15] and involves phenotypic and reversible transitions that have been categorized into at least 4 stages according to Kreutzberg [16] (see Figure 1). Microglial activation is a patho-physiological feature of many brain diseases and for many years its key function was thought to be solely the removal of cellular debris [13]. Overwhelming evidence now shows that microglial activation is a phenomenon actively involved in neurodegeneration or neuroprotection (reviewed in $[13,17]$ ).

Despite the many differences among animal models of PD and among PD patients, a common feature found in the $\mathrm{SN}$ in $\mathrm{PD}$ is the presence of microglial activation (reviewed in $[13,17-20])$. Since the first description of microglial activation in the SN of PD brains by McGeer and colleagues in 1988 [21], numerous studies (more than 30) have repeated this observation in animal models and PD patients (reviewed in $[13,19])$. Microglial-secreted factors that are associated with PD pathology include Interleukin-(IL) $1 \beta$, Tumor necrosis Factor $\alpha$ (TNF), IL-6, IL-2, Interferon- $\gamma$ (IFN- $\gamma$ ), prostaglandins, and reactive oxygen and nitrogen species (for a comprehensive review see [22]). This is unlike astrogliosis, which is not as pronounced nor so consistently present in $\mathrm{PD}$ patients or animal models $[23,24]$. Therefore, the discussion will be focused on microglial activation. The role of astrocytes in PD has been recently discussed in [19].

\section{Microglial Activation and PD-Animal Models}

In the 6-OHDA model of nigrostriatal neurodegeneration, microglial activation in the SN was morphological defined as stage II and III, but not IV [24], see Figure 1. During the neurodegenerative process, transcription but not translation of key proinflammatory cytokines, was markedly increased [24]. In this way no proinflammatory environment was generated as a consequence of neuronal cell death. Therefore, microglial activation during neurodegeneration is not associated with the production of a proinflammatory milieu, as previously presupposed $[8,24]$. This observation concurs with the physiological role of macrophages during the clearance of apoptotic cells in the periphery where such macrophage activation does not promote inflammation [25]. An example of this would be the clearance of neutrophils: it is estimated that $10^{10}$ neutrophils/day enter apoptosis and that macrophages are responsible for removing them in humans. Were this process to be proinflammatory, the human body would be permanently inflamed. In PD, most if not all neuronal loss in the $\mathrm{SN}$ is supposed to be apoptotic [26] and thus, even though activated microglia are essential to remove neuronal cell debris, a proinflammatory milieu should not be expected from this activation.

Activated microglial cells with higher proinflammatory cytokine mRNA but not protein expression have been described as being in a "primed" state, ready to produce an outburst of proinflammatory cytokines if a second stimulus appears (see Figure 1). Indeed, it has been demonstrated that if a subtoxic dose of a proinflammatory stimulus, such as bacterial endotoxin, is delivered to the degenerating $\mathrm{SN}$, the translation of increased levels of mRNA coding for IL-1 takes place and an intense proinflammatory environment is generated [8]. Interestingly, this effect can also be elicited systemically by the sustained expression of circulating IL-1 [8]. Of utmost importance was the observation that this displacement of the equilibrium towards a proinflammatory milieu in the $\mathrm{SN}$ exacerbated disease progression and triggered earlier and more pronounced motor signs [8] (see Figure 1). Reversing the order of the stimuli could lead to a similar or a different observation. It has been described that previous exposure to LPS rendered the animals more susceptible to the neurotoxic effects of 6OHDA [27]. On the other hand, the prior inoculation of IL-1 has a neuroprotective effects on the nigral neurons [28]. In addition, if this preexposition to inflammation was performed during pregnancy, the adult offspring were not only more susceptible to 6-OHDA administration, but had fewer dopaminergic cells in the $\mathrm{SN}$ at postnatal day 10 compared to controls $[29,30]$.

Neurons in the SN have been shown to be particularly susceptible to microglial-mediated toxicity in vitro and in vivo $[6,7]$, and anti-inflammatory interventions have been shown to be neuroprotective in animal models of PD [31-35]. By contrast, some early work has reported neuroprotective effects of inflammatory mediators. Variables such as duration and amount of expression of a specific cytokine seem to be important to anticipate the final effect of a given cytokine on neuronal viability. For example, the acute injection of IL- $1 \beta$ in the SN was not toxic for dopaminergic neurons in vivo if the cytokine was injected alone (10 ng or 1000 Units) or in combination with 1000 Units of TNF and 100 Units of IFN- $\gamma$ in the SN $[36,37]$. If, however, the expression of IL-1 or TNF in the SN was sustained between 14 and 21 days, it caused neuronal death, motor symptoms, and microglial activation to Stage IV [38-40]. Similarly, long-term inhibition of IL-1 or TNF attenuated loss of dopaminergic neurons in PD models [8, 41-43].

It can be concluded from these data that dosage and duration of expression are important to predict an effect of 


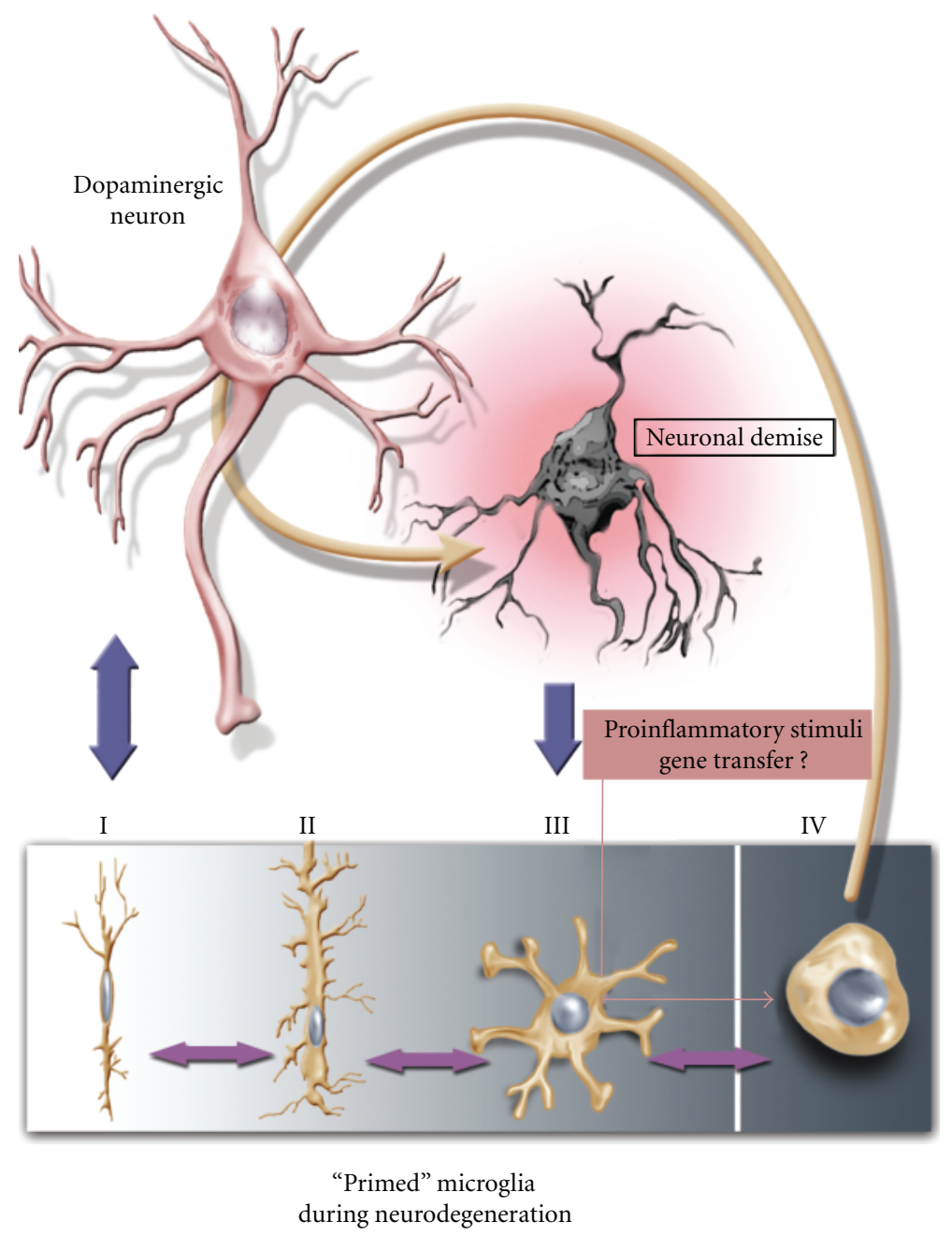

FIGURE 1: Schematic representation of the interactions between microglial activation and gene transfer. During neuronal death, microglial cells are activated to a "primed" state, where no proinflammatory cytokines are secreted. After receiving an additional (proinflammatory) stimulus, microglia activation changes into an exacerbated proinflammatory state that leads to increased neurodegeneration. Depending on variables such as type and dosage of vector used, inoculation of viral vectors might provide this second proinflammatory stimulus.

a given cytokine on neuronal viability in the SN. However, some univocal effects could be defined. For example, if a subtoxic inflammatory stimulus is present in the degenerating SN where "primed" microglia are present, neuronal death could be exacerbated. Similarly, if the expression of IL-1 or TNF in the SN at proinflammatory levels is sustained, neuronal cell death is likely to occur.

\section{Microglial Activation and PD-Clinical Data}

As explained above, microglial activation has been found in the SN of PD patients (reviewed in $[13,19]$ ) in postmortem tissue sample and also by noninvasive imaging [20,44]. In addition, higher expression levels of IL-1, IL-2, IL-6, and TNF were found in striatal postmortem samples of PD patients [45-47]. Using the peripheral benzodiazepine receptor as ligand (PK-11195) in a positron emission tomography study, neuroinflammatory processes have been verified in the pons, basal ganglia, and frontal cortex of PD patients [44].
The resolution of this technique has not allowed an accurate study of the SN. It should also be noted that evidence from PD patients indicates that microglial activation is not restricted to the $\mathrm{SN}$, but it can also involve the putamen, hippocampus, brain stem, and cingulate and temporal cortex $[44,48]$.

\section{Possible Effects of Inflammation-Eliciting Gene Transfer on the Degenerating SN}

Viral gene delivery will introduce an antigenic load into the brain [49]. These antigens will invariably elicit at least, a transient innate immune response [49]. As stated before, in a naïve CNS, the innate immune response can be dissociated from the adaptive immune response in the brain parenchyma; for example, the systemic immune system can remain ignorant of a first antigenic challenge into the brain parenchyma. However, what happens if this viral load hits a brain region in which on-going inflammation or "primed" 
microglial activation (as most likely will occur in the SN of PD patients) is present? Several variables need to be considered to answer this complex question, including vector type, vector dosage, method of delivery, patients' age, stage of disease progression, and transgene used.

5.1. Vector Type and Dosage. The three main types of vector that emerge as solid candidates for gene therapy clinical trials for PD are adenoviral, adeno-associated, and lentiviral vectors.

Adenoviral vectors are among the most studied vectors for gene delivery in the CNS. In particular, the immune response to this virus in the brain has been extensively investigated. First-generation adenoviral vectors (Fg-Ad) allow transgene expression in the naïve brain for up to one year, which appears to be independent of a transient and initial inflammatory response $[8,12,38,39,49-53]$. However, preexisting or subsequent systemic immune response to adenovirus in the host abolish, or at least reduce by half, the transgene expression and can lead to cytotoxicity ([49-52], reviewed in $[5,11])$. Direct inoculation of $5 \times 10^{6}-1 \times 10^{7}$ infective particles of control adenovectors in the $\mathrm{SN}$, caused between $10-25 \%$ of neurodegeneration per se $[39,40]$, reenforcing the idea of an increased susceptibility of this region to inflammation. As is the case with any viral vector, dosage determines the response detected after vector administration. A threshold of more than $10^{7}$ infective particles of adenoviral vectors in the periphery was determined to be needed to eliminate transgene expression in the brain [52]. This drawback is not seen when high capacity adenoviral vectors (hc-Ad) are used for gene transfer in the CNS. Therefore, hcAd seem to be better vectors for gene transfer in the brain than Fg-Ad. Nevertheless, the viral capsid is identical for both vectors and will elicit a response that, within a degenerating $\mathrm{SN}$, may cause an increase in inflammation with toxic effects. In addition, internalized adenoviral DNA activates an inflammasome-dependent maturation of proIL- 1 to the active form of IL-1 in macrophages. Thus, it is not unlikely that any type of adenoviral vector injected in the degenerating SN will contribute to drive the environment to a proinflammatory milieu [54].

Adeno-associated vectors (AAV) are the vectors of choice for the vast majority of gene therapy clinical trials against PD [4]. The intrastriatal injection of relatively low titers of AAV $\left(2-4 \times 10^{8}\right.$ i.p. $)$ in naïve animals provokes a low innate immune response with no mononuclear cell infiltrate or cuffing of nearby blood vessels $[55,56]$. However, at higher doses, a transient but significant astrogliosis can be detected [57]. In addition, in animal models previously exposed to systemic AAV, immunization inhibited AAV serotype 2 (AAV-2) gene transfer in the CNS [56], and readministration of AAV in the brain induced a greater inflammatory response $[56,58]$.

Results from Phase I and II clinical trials for PD have not reported severe adverse effects related to AAV administration [59-63]. Gene transfer was performed in the putamen or in the subthalamic nucleus. Despite these encouraging results, long-term analysis of a bigger cohort is needed to provide robust data on the degree of safety of these vectors during gene transfer in the brain. Unfortunately, the possible toxic effects of inflammation that could mask the potential beneficial effect of the treatment were apparently not studied. Recently, a Phase I/II clinical trial has been approved to inoculate patients with AAV vectors expressing neurturin not only in the putamen, but also in the SN (http://www.ClinicalTrials.gov identifier: NCT00985517). According to the data on the susceptibility of the $\mathrm{SN}$ to inflammation discussed above, it will be valuable to study the inflammatory response to the treatment in each patient to draw conclusions on safety and possibly efficacy in this trial.

Lentiviral vectors have been approved as vehicles for clinical gene transfer of aromatic amino-acid decarboxylase, Tyrosine Hydroxylase (TH) and GTP-cyclohydrolase 1, all three genes necessary for dopamine synthesis [64]. Stimulation of dendritic cells by lentiviral vectors is weak compared with other single-stranded RNA viruses [65]. In addition, a reduced immune response has been detected after brain administration of multiple-deleted lentiviral vectors [66]. Worryingly, time-and dose-dependent downregulation of $\mathrm{TH}$, the rate limiting enzyme in dopamine synthesis, has been reported after lentiviral-delivery of neurturin [67]. In addition, in the periphery, lentiviral delivery of reporter genes into the lung triggered T-cell mediated immune responses against the transgene [68]. Results from the abovementioned clinical trial are awaited to verify the seemingly low-level immune response against lentiviral vectors in the brain. Finally, the phenomenon of gene silencing that depends of vector and promoter used and state of differentiation of the target cell should be considered [69]. For example, it could be tempting to try to compensate loss of expression by gene silencing with increased dosage of vector administered, increasing the probability of eliciting a proinflammatory response and therefore toxic effects on the SN.

5.2. Method of Gene Delivery. Independently of the vector of choice, the method of gene delivery may dramatically influence the magnitude and characteristics of the immune reaction against the gene delivery. For example, it is crucial that the method of vector delivery is accurate enough to prevent antigens from reaching the brain ventricular system and the deep cervical lymph nodes so as to keep the systemic immune system ignorant of the antigenic challenge produced by gene delivery in the CNS [5]. If this cannot be achieved, a systemic immune reaction against the viral vector and/or transgene delivered is likely to be generated [5]. At the very least, it will foreshorten the temporal expression profile and, at worst it will promote neurotoxic immune-mediated effects in the brain (reviewed in [11]). In conclusion, a delivery method of high accuracy is needed to prevent antigen diffusion into undesired brain regions.

5.3. Age and Disease Progression. In parallel, age-related changes in immune reactivity include enhanced BloodBrain Barrier permeability and increased microglial and astroglial reactivity [70-72]. Therefore, changes in the 
immune response against viral gene transfer can be expected in a manner that is dependent on the age of the treated subject.

L-Dopa therapy is quite effective in the early stages of most PD patients. Therefore, most clinical trials, including those related to gene therapy, are usually target to latestage PD patients. It has been proposed that neuroprotective strategies will not be beneficial to these patients since at that stage of the disease there are limited amounts of dopaminergic neurons to protect. Likewise, late-stage PD patients will have encountered more opportunities for neuroinflammation in the SN to start and therefore have a higher risk of vector-mediated toxicity by gene transfer in the SN.

5.4. Transgene Used. Immune responses against the transgene are not infrequent and depend on whether there has been a prior exposure to it and whether it is syn- or xenogenic to the host [11]. In addition, it should also be borne in mind that chronic inflammation can facilitate dendritic cell infiltration into the CNS, which can facilitate antigen presentation to naïve T cells [73]. Again this is a plausible situation in the degenerating $\mathrm{SN}$.

\section{How Can All These Risks Be Minimized?}

A better understanding of the immunological component of the SN in PD patients together with studies on the possible beneficial effects of complementary anti-inflammatory treatments, changes in vector serotype, novel chemical formulations, and novel vector design will all help to design the best scenario to avoid undesired effects of an inflammatory response to gene delivery in the CNS. Alternatively, taking advantage of certain intrinsic properties of viral vectors might help to circumvent the risk of inflammation in the SN. For example, vectors can be used for the retrograde delivery of genes (e.g., adenoviral vectors could be administered in the striatal terminals of nigral neurons to deliver genes in the SN [38]). Certainly, this strategy has the disadvantage that it can reduce the amount of transgene delivered to the $\mathrm{SN}$ as seems to be the case in the Phase II trial with AAV-neurturin [63]. Nevertheless, it is a useful strategy to be considered when planning future gene therapy strategies using different vectors or transgenes. In addition, analyzing risk factors for each treatment and patient (age, method of gene delivery, immunogenicity of vector used, immunological status of the brain area to treat, influence of the transgene to be transferred, dosage, previous exposure to the virus used as vector) will certainly reduce the risk of immune-derived toxicity during gene transfer protocols against PD. In the future, this immunological risk analysis could even be used as an inclusion or exclusion criterium. Unfortunately, nowadays knowledge is still lacking to define parameters with univocal effects on the immunological response of gene transfer into the $\mathrm{SN}$, and technology is behind to determine the immunological status of the SN at the time of gene transfer. Therefore, the most reasonable measurement is to design a clinical trial protocol to reduce the risk of inflammation-mediated toxicity as much as possible.
For example, we would like to propose that if a constellation of risk factors (increased age, late stage of disease, previous exposed to the virus, high viral dose) is present, an anti-inflammatory therapy could be considered [44]. Anti-inflammatory treatments such as COX-2 inhibition, minocycline, and naloxone have promising effects on animal models of PD [31-35]. In the context of a possible inflammatory reaction in a gene therapy protocol in the $\mathrm{SN}$, these anti-inflammatory treatments may be reconsidered as complementary treatments. In addition, not only conventional anti-inflammatory therapies could be helpful to reduce the inflammatory risk of a PD patient, but antiinflammatory molecules could be delivered by gene transfer in addition to other therapeutic genes. In particular, the viral delivery of Interleukin-10 or IL-1ra has been shown to be neuroprotective in the 6-OHDA rat model of $\mathrm{PD}[8,74]$.

\section{Conclusions}

The SN is the main area of neurodegeneration in PD. Microglial activation and proinflammatory cytokine production have special characteristics in the degenerating $\mathrm{SN}$ that should not be underestimated when designing a gene transfer protocol in that area. It is expected that "primed" microglia or an on-going inflammatory response will be present at the time of gene transfer in PD patients. Numerous variables are in play that could change the expected outcome of gene delivery. An exhaustive analysis of the status of risks factors known to lead to inflammation at the moment of clinical intervention, leading to complementary anti-inflammatory treatments and/or alternative gene delivery strategies or additional genes delivered (e.g., IL-10) is proposed to reduce undesired, inflammation-driven side effects. Gene therapy against PD has reached maturity with eight clinical trials approved. It is of importance to consider, with the limitations of the available technology and knowledge, all variables affecting the immunological status of the PD patient and the possible interactions with the inflammatory component of gene delivery. This analysis should increase the probability of providing safe gene transfer in the $\mathrm{SN}$ and reduce inflammation-biased results that can obscure the efficacy of a given gene transfer protocol.

\section{Acknowledgments}

The authors wish to thank Dr. Daniel Anthony for useful suggestions and critical reading of the paper and Estela Lafita for her drawings. Fernando Pitossi is recipient of grants from the Agencia Nacional de Promoción Científica y Tecnológica de Argentina, Pfizer Inc. and the René Barón Foundation. Fernando Pitossi is member of the research career of CONICET.

\section{References}

[1] T. Hatano, S. I. Kubo, S. Sato, and N. Hattori, "Pathogenesis of familial Parkinson's disease: new insights based on monogenic forms of Parkinson's disease," Journal of Neurochemistry, vol. 111, no. 5, pp. 1075-1093, 2009. 
[2] A. E. Lang and A. M. Lozano, "Parkinson's disease: second of two parts," The New England Journal of Medicine, vol. 339, no. 16, pp. 1130-1143, 1998.

[3] A. L. Benabid, “Gene therapy for Parkinson's disease: do we have the cure?" Lancet Neurology, vol. 9, no. 12, pp. 1142-1143, 2010.

[4] M. M. McMenamin and M. J. A. Wood, "Progress and prospects: immunobiology of gene therapy for neurodegenerative disease: Prospects and risks," Gene Therapy, vol. 17, no. 4, pp. 448-458, 2010.

[5] P. R. Lowenstein, K. Kroeger, and M. G. Castro, "Immunology of neurological gene therapy: how $\mathrm{T}$ cells modulate viral vector-mediated therapeutic transgene expression through immunological synapses," Neurotherapeutics, vol. 4, no. 4, pp. 715-724, 2007.

[6] W. G. Kim, R. P. Mohney, B. Wilson, G. H. Jeohn, B. Liu, and J. S. Hong, "Regional difference in susceptibility to lipopolysaccharide-induced neurotoxicity in the rat brain: role of microglia," Journal of Neuroscience, vol. 20, no. 16, pp. 6309 6316, 2000.

[7] A. Castaño, A. J. Herrera, J. Cano, and A. Machado, "Lipopolysaccharide intranigral injection induces inflammatory reaction and damage in nigrostriatal dopaminergic system," Journal of Neurochemistry, vol. 70, no. 4, pp. 15841592, 1998.

[8] M. C. P. Godoy, R. Tarelli, C. C. Ferrari, M. I. Sarchi, and F. J. Pitossi, "Central and systemic IL-1 exacerbates neurodegeneration and motor symptoms in a model of Parkinson's disease," Brain, vol. 131, no. 7, pp. 1880-1894, 2008.

[9] V. H. Perry, "A revised view of the central nervous system microenvironment and major histocompatibility complex class II antigen presentation," Journal of Neuroimmunology, vol. 90, no. 2, pp. 113-121, 1998.

[10] V. H. Perry, M. D. Bell, H. C. Brown, and M. K. Matyszak, "Inflammation in the nervous system," Current Opinion in Neurobiology, vol. 5, no. 5, pp. 636-641, 1995.

[11] P. R. Lowenstein, R. J. Mandel, W. D. Xiong, K. Kroeger, and M. G. Castro, "Immune responses to adenovirus and adenoassociated vectors used for gene therapy of brain diseases: the role of immunological synapses in understanding the cell biology of neuroimmune interactions," Current Gene Therapy, vol. 7, no. 5, pp. 347-360, 2007.

[12] P. R. Lowenstein and M. G. Castro, "Inflammation and adaptive immune responses to adenoviral vectors injected into the brain: peculiarities, mechanisms, and consequences," Gene Therapy, vol. 10, no. 11, pp. 946-954, 2003.

[13] V. H. Perry, J. A. R. Nicoll, and C. Holmes, "Microglia in neurodegenerative disease," Nature Reviews Neurology, vol. 6, no. 4, pp. 193-201, 2010.

[14] M. J. Carson, T. V. Bilousova, S. S. Puntambekar, B. Melchior, J. M. Doose, and I. M. Ethell, "A rose by any other name? The potential consequences of microglial heterogeneity during CNS health and disease," Neurotherapeutics, vol. 4, no. 4, pp. 571-579, 2007.

[15] C. A. Colton, "Heterogeneity of microglial activation in the innate immune response in the brain," Journal of Neuroimmune Pharmacology, vol. 4, no. 4, pp. 399-418, 2009.

[16] G. W. Kreutzberg, "Microglia: a sensor for pathological events in the CNS," Trends in Neurosciences, vol. 19, no. 8, pp. 312318, 1996.

[17] C. K. Glass, K. Saijo, B. Winner, M. C. Marchetto, and F. H. Gage, "Mechanisms underlying inflammation in neurodegeneration," Cell, vol. 140, no. 6, pp. 918-934, 2010.
[18] C. M. Long-Smith, A. M. Sullivan, and Y. M. Nolan, "The influence of microglia on the pathogenesis of Parkinson's disease," Progress in Neurobiology, vol. 89, no. 3, pp. 277-287, 2009.

[19] E. C. Hirsch and S. Hunot, "Neuroinflammation in Parkinson's disease: a target for neuroprotection?" The Lancet Neurology, vol. 8, no. 4, pp. 382-397, 2009.

[20] Y. Ouchi, S. Yagi, M. Yokokura, and M. Sakamoto, "Neuroinflammation in the living brain of Parkinson's disease," Parkinsonism and Related Disorders, vol. 15, supplement 3, pp. S200-S204, 2009.

[21] P. L. McGeer, S. Itagaki, B. E. Boyes, and E. G. McGeer, "Reactive microglia are positive for HLA-DR in the substantia nigra of Parkinson's and Alzheimer's disease brains," Neurology, vol. 38, no. 8, pp. 1285-1291, 1988.

[22] M. G. Tansey and M. S. Goldberg, "Neuroinflammation in Parkinson's disease: its role in neuronal death and implications for therapeutic intervention," Neurobiology of Disease, vol. 37, no. 3, pp. 510-518, 2010.

[23] B. Mirza, H. Hadberg, P. Thomsen, and T. Moos, "The absence of reactive astrocytosis is indicative of a unique inflammatory process in Parkinson's disease," Neuroscience, vol. 95, no. 2, pp. 425-432, 1999.

[24] A. M. Depino, C. Earl, E. Kaczmarczyk et al., "Microglial activation with atypical proinflammatory cytokine expression in a rat model of Parkinson's disease," European Journal of Neuroscience, vol. 18, no. 10, pp. 2731-2742, 2003.

[25] V. A. Fadok, D. L. Bratton, A. Konowal, P. W. Freed, J. Y. Westcott, and P. M. Henson, "Macrophages that have ingested apoptotic cells in vitro inhibit proinflammatory cytokine production through autocrine/paracrine mechanisms involving TGF-beta, PGE2, and PAF," Journal of Clinical Investigation, vol. 110, no. 4, pp. 890-898, 1998.

[26] Y. He, T. Lee, and S. K. Leong, "6-Hydroxydopamine induced apoptosis of dopaminergic cells in the rat substantia nigra," Brain Research, vol. 858, no. 1, pp. 163-166, 2000.

[27] J. B. Koprich, C. Reske-Nielsen, P. Mithal, and O. Isacson, "Neuroinflammation mediated by IL- $1 \beta$ increases susceptibility of dopamine neurons to degeneration in an animal model of Parkinson's disease," Journal of Neuroinflammation, vol. 5, article 8, 2008.

[28] J. Saura, M. Parés, J. Bové et al., "Intranigral infusion of interleukin- $1 \beta$ activates astrocytes and protects from subsequent 6-hydroxydopamine neurotoxicity," Journal of Neurochemistry, vol. 85, no. 3, pp. 651-661, 2003.

[29] Z. D. Ling, D. A. Gayle, S. Y. Ma et al., "In utero bacterial endotoxin exposure causes loss of tyrosine hydroxylase neurons in the postnatal rat midbrain," Movement Disorders, vol. 17, no. 1, pp. 116-124, 2002.

[30] P. M. Carvey, Q. Chang, J. W. Lipton, and Z. Ling, "Prenatal exposure to the bacteriotoxin lipopolysaccharide leads to longterm losses of dopamine neurons in offspring: a potential, new model of Parkinson's disease," Frontiers in Bioscience, vol. 8, pp. s826-s837, 2003.

[31] Y. He, S. Appel, and W. Le, "Minocycline inhibits microglial activation and protects nigral cells after 6-hydroxydopamine injection into mouse striatum," Brain Research, vol. 909, no. 1-2, pp. 187-193, 2001.

[32] A. Hald and J. Lotharius, "Oxidative stress and inflammation in Parkinson's disease: is there a causal link?" Experimental Neurology, vol. 193, no. 2, pp. 279-290, 2005.

[33] R. Sánchez-Pernaute, A. Ferree, O. Cooper, M. Yu, A. L. Brownell, and O. Isacson, "Selective COX-2 inhibition prevents progressive dopamine neuron degeneration in a rat 
model of Parkinson's disease," Journal of Neuroinflammation, vol. 1, article 6, 2004.

[34] D. C. Wu, V. Jackson-Lewis, M. Vila et al., "Blockade of microglial activation is neuroprotective in the 1-methyl-4phenyl-1,2,3,6-tetrahydropyridine mouse model of Parkinson disease," Journal of Neuroscience, vol. 22, no. 5, pp. 1763-1771, 2002.

[35] B. Liu, L. Du, and J. S. Hong, "Naloxone protects rat dopaminergic neurons against inflammatory damage through inhibition of microglia activation and superoxide generation," Journal of Pharmacology and Experimental Therapeutics, vol. 293, no. 2, pp. 607-617, 2000.

[36] A. Castaño, A. J. Herrera, J. Cano, and A. Machado, "The degenerative effect of a single intranigral injection of LPS on the dopaminergic system is prevented by dexamethasone, and not mimicked by rh-TNF- $\alpha$ IL- $1 \beta$ IFN- $\gamma$," Journal of Neurochemistry, vol. 81, no. 1, pp. 150-157, 2002.

[37] A. Depino, C. Ferrari, M. C. Pott Godoy, R. Tarelli, and F. J. Pitossi, "Differential effects of interleukin- $1 \beta$ on neurotoxicity, cytokine induction and glial reaction in specific brain regions," Journal of Neuroimmunology, vol. 168, no. 1-2, pp. 96-110, 2005.

[38] M. C. Pott Godoy, C. C. Ferrari, and F. J. Pitossi, "Nigral neurodegeneration triggered by striatal AdIL- 1 administration can be exacerbated by systemic IL-1 expression," Journal of Neuroimmunology, vol. 222, no. 1-2, pp. 29-39, 2010.

[39] A. L. De Lella Ezcurra, M. Chertoff, C. Ferrari, M. Graciarena, and F. Pitossi, "Chronic expression of low levels of tumor necrosis factor- $\alpha$ in the substantia nigra elicits progressive neurodegeneration, delayed motor symptoms and microglia/macrophage activation," Neurobiology of Disease, vol. 37, no. 3, pp. 630-640, 2010.

[40] C. C. Ferrari, M. C. Pott Godoy, R. Tarelli, M. Chertoff, A. M. Depino, and F. J. Pitossi, "Progressive neurodegeneration and motor disabilities induced by chronic expression of IL- $1 \beta$ in the substantia nigra," Neurobiology of Disease, vol. 24, no. 1, pp. 183-193, 2006.

[41] T. A. Tran, M. K. McCoy, M. B. Sporn, and M. G. Tansey, "The synthetic triterpenoid CDDO-methyl ester modulates microglial activities, inhibits TNF production, and provides dopaminergic neuroprotection," Journal of Neuroinflammation, vol. 5, article 14, 2008.

[42] M. K. McCoy, T. N. Martinez, K. A. Ruhn et al., "Blocking soluble tumor necrosis factor signaling with dominantnegative tumor necrosis factor inhibitor attenuates loss of dopaminergic neurons in models of Parkinson's disease," Journal of Neuroscience, vol. 26, no. 37, pp. 9365-9375, 2006.

[43] M. K. McCoy, K. A. Ruhn, T. N. Martinez, F. E. McAlpine, A. Blesch, and M. G. Tansey, "Intranigral lentiviral delivery of dominant-negative TNF attenuates neurodegeneration and behavioral deficits in hemiparkinsonian rats," Molecular Therapy, vol. 16, no. 9, pp. 1572-1579, 2008.

[44] A. Gerhard, N. Pavese, G. Hotton et al., "In vivo imaging of microglial activation with [C](R)-PK11195 PET in idiopathic Parkinson's disease," Neurobiology of Disease, vol. 21, no. 2, pp. 404-412, 2006.

[45] M. Mogi, M. Harada, T. Kondob et al., "Interleukin-1 $\beta$, interleukin-6, epidermal growth factor and transforming growth factor- $\alpha$ are elevated in the brain from parkinsonian patients," Neuroscience Letters, vol. 180, no. 2, pp. 147-150, 1994.

[46] M. Mogi, M. Harada, P. Riederer, H. Narabayashi, K. Fujita, and T. Nagatsu, "Tumor necrosis factor- $\alpha$ (TNF- $\alpha$ ) increases both in the brain and in the cerebrospinal fluid from parkinsonian patients," Neuroscience Letters, vol. 165, no. 1-2, pp. 208-210, 1994.

[47] M. Mogi, M. Harada, T. Kondo, P. Riederer, and T. Nagatsu, "Interleukin-2 but not basic fibroblast growth factor is elevated in parkinsonian brain. Short communication," Journal of Neural Transmision, vol. 103, no. 8-9, pp. 1077-1081, 1996.

[48] K. Imamura, N. Hishikawa, M. Sawada, T. Nagatsu, M. Yoshida, and Y. Hashizume, "Distribution of major histocompatibility complex class II-positive microglia and cytokine profile of Parkinson's disease brains," Acta Neuropathologica, vol. 106, no. 6, pp. 518-526, 2003.

[49] C. Barcia, M. Jimenez-Dalmaroni, K. M. Kroeger et al., "Oneyear expression from high-capacity adenoviral vectors in the brains of animals with pre-existing anti-adenoviral immunity: clinical implications," Molecular Therapy, vol. 15, no. 12, pp. 2154-2163, 2007.

[50] C. E. Thomas, G. Schiedner, S. Kochanek, M. G. Castro, and P. R. Löwenstein, "Peripheral infection with adenovirus causes unexpected long-term brain inflammation in animals injected intracranially with first-generation, but not with high-capacity, adenovirus vectors: toward realistic long-term neurological gene therapy for chronic diseases," Proceedings of the National Academy of Sciences of the United States of America, vol. 97, no. 13, pp. 7482-7487, 2000.

[51] C. E. Thomas, G. Schiedner, S. Kochanek, M. G. Castro, and P. R. Lowenstein, "Preexisting antiadenoviral immunity is not a barrier to efficient and stable transduction of the brain, mediated by novel high-capacity adenovirus vectors," Human Gene Therapy, vol. 12, no. 7, pp. 839-846, 2001.

[52] C. Barcia, C. Gerdes, W. D. Xiong et al., "mmunological thresholds in neurological gene therapy: highly efficient elimination of transduced cells might be related to the specific formation of immunological synapses between $\mathrm{T}$ cells and virus-infected brain cells," Neuron Glia Biology, vol. 2, no. 4, pp. 309-322, 2006.

[53] C. C. Ferrari, A. M. Depino, F. Prada et al., "Reversible demyelination, blood-brain barrier breakdown, and pronounced neutrophil recruitment induced by chronic IL-1 expression in the brain," American Journal of Pathology, vol. 165, no. 5, pp. 1827-1837, 2004.

[54] D. A. Muruve, V. Pétrilli, A. K. Zaiss et al., "The inflammasome recognizes cytosolic microbial and host DNA and triggers an innate immune response," Nature, vol. 452, no. 7183, pp. 103$107,2008$.

[55] R. J. Mandel, K. G. Rendahl, S. K. Spratt, R. O. Snyder, L. K. Cohen, and S. E. Leff, "Characterization of intrastriatal recombinant adeno-associated virus- mediated gene transfer of human tyrosine hydroxylase and human GTP- cyclohydrolase I in a rat model of Parkinson's disease," Journal of Neuroscience, vol. 18, no. 11, pp. 4271-4284, 1998.

[56] C. S. Peden, C. Burger, N. Muzyczka, and R. J. Mandel, "Circulating anti-wild-type adeno-associated virus type 2 (AAV2) antibodies inhibit recombinant AAV2 (rAAV2)-mediated, but not rAAV5-mediated, gene transfer in the brain," Journal of Virology, vol. 78, no. 12, pp. 6344-6359, 2004.

[57] S. Reimsnider, F. P. Manfredsson, N. Muzyczka, and R. J. Mandel, "Time course of transgene expression after intrastriatal pseudotyped rAAV2/1, rAAV2/2, rAAV2/5, and rAAV2/8 transduction in the rat," Molecular Therapy, vol. 15, no. 8, pp. 1504-1511, 2007.

[58] M. Y. Mastakov, K. Baer, C. W. Symes, C. B. Leichtlein, R. M. Kotin, and M. J. During, "Immunological aspects of recombinant adeno-associated virus delivery to the mammalian 
brain," Journal of Virology, vol. 76, no. 16, pp. 8446-8454, 2002.

[59] J. L. Eberling, W. J. Jagust, C. W. Christine et al., "Results from a phase I safety trial of hAADC gene therapy for Parkinson disease," Neurology, vol. 70, no. 21, pp. 1980-1983, 2008.

[60] W. J. Marks, J. L. Ostrem, L. Verhagen et al., "Safety and tolerability of intraputaminal delivery of CERE-120 (adenoassociated virus serotype 2-neurturin) to patients with idiopathic Parkinson's disease: an open-label, phase I trial," The Lancet Neurology, vol. 7, no. 5, pp. 400-408, 2008.

[61] M. G. Kaplitt, A. Feigin, C. Tang et al., "Safety and tolerability of gene therapy with an adeno-associated virus (AAV) borne GAD gene for Parkinson's disease: an open label, phase I trial," Lancet, vol. 369, no. 9579, pp. 2097-2105, 2007.

[62] A. Feigin, M. G. Kaplitt, C. Tang et al., "Modulation of metabolic brain networks after subthalamic gene therapy for Parkinson's disease," Proceedings of the National Academy of Sciences of the United States of America, vol. 104, no. 49, pp. 19559-19564, 2007.

[63] W. J. Marks Jr., R. T. Bartus, J. Siffert et al., "Gene delivery of AAV2-neurturin for Parkinson's disease: a double-blind, randomised, controlled trial," The Lancet Neurology, vol. 9, no. 12, pp. 1164-1172, 2010.

[64] B. Jarraya, S. Boulet, G. S. Ralph et al., "Dopamine gene therapy for Parkinson's disease in a nonhuman primate without associated dyskinesia," Science Translational Medicine, vol. 1, no. 2, p. 2ra4, 2009.

[65] A. Pichlmair, S. S. Diebold, S. Gschmeissner et al., "Tubulovesicular structures within Vesicular stomatitis virus G proteinpseudotyped lentiviral vector preparations carry DNA and stimulate antiviral responses via toll-like receptor 9," Journal of Virology, vol. 81, no. 2, pp. 539-547, 2007.

[66] C. F. Valori, K. Ning, M. Wyles, and M. Azzouz, "Development and applications of non-HIV-based lentiviral vectors in neurological disorders," Current Gene Therapy, vol. 8, no. 6, pp. 406-418, 2008.

[67] B. Georgievska, D. Kirik, and A. Björklund, "Overexpression of glial cell line-derived neurotrophic factor using a lentiviral vector induces time- and dose-dependent downregulation of tyrosine hydroxylase in the intact nigrostriatal dopamine system," Journal of Neuroscience, vol. 24, no. 29, pp. 64376445, 2004.

[68] M. P. Limberis, C. L. Bell, J. Heath, and J. M. Wilson, "Activation of transgene-specific $\mathrm{T}$ cells following lentivirusmediated gene delivery to mouse lung," Molecular Therapy, vol. 18, no. 1, pp. 143-150, 2010.

[69] M. Vroemen, N. Weidner, and A. Blesch, "Loss of gene expression in lentivirus- and retrovirus-transduced neural progenitor cells is correlated to migration and differentiation in the adult spinal cord," Experimental Neurology, vol. 195, no. 1, pp. 127-139, 2005.

[70] S. Sugama, L. Yang, B. P. Cho et al., "Age-related microglial activation in 1-methyl-4-phenyl-1,2,3,6-tetrahydropyridine (MPTP)-induced dopaminergic neurodegeneration in C57BL/6 mice," Brain Research, vol. 964, no. 2, pp. 288-294, 2003.

[71] H. Y. Chung, H. J. Kim, J. W. Kim, and B. P. Yu, “The inflammation hypothesis of aging: molecular modulation by calorie restriction," Annals of the New York Academy of Sciences, vol. 928, pp. 327-335, 2001.

[72] C. Pelegrí, A. M. Canudas, J. del Valle et al., "Increased permeability of blood-brain barrier on the hippocampus of a murine model of senescence," Mechanisms of Ageing and Development, vol. 128, no. 9, pp. 522-528, 2007.
[73] E. J. McMahon, S. L. Bailey, C. V. Castenada, H. Waldner, and S. D. Miller, "Epitope spreading initiates in the CNS in two mouse models of multiple sclerosis," Nature Medicine, vol. 11, no. 3, pp. 335-339, 2005.

[74] L. C. Johnston, X. Su, K. Maguire-Zeiss et al., "Human interleukin-10 gene transfer is protective in a rat model of parkinson's disease," Molecular Therapy, vol. 16, no. 8, pp. 1392-1399, 2008. 


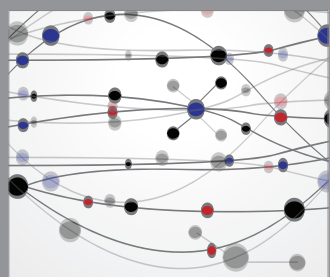

The Scientific World Journal
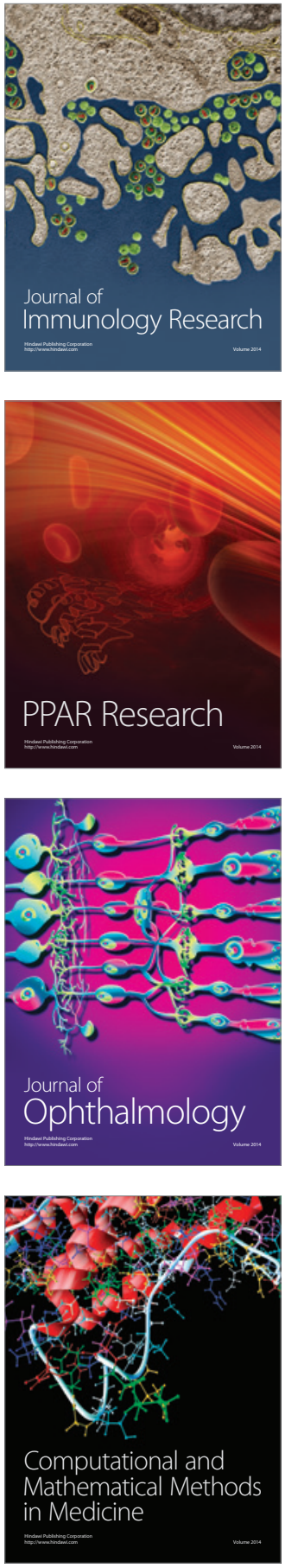

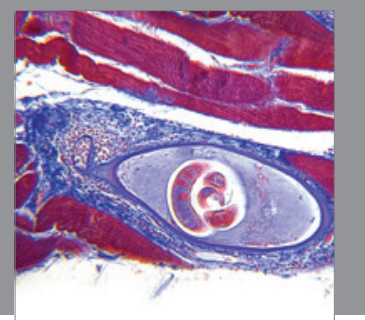

Gastroenterology

Research and Practice
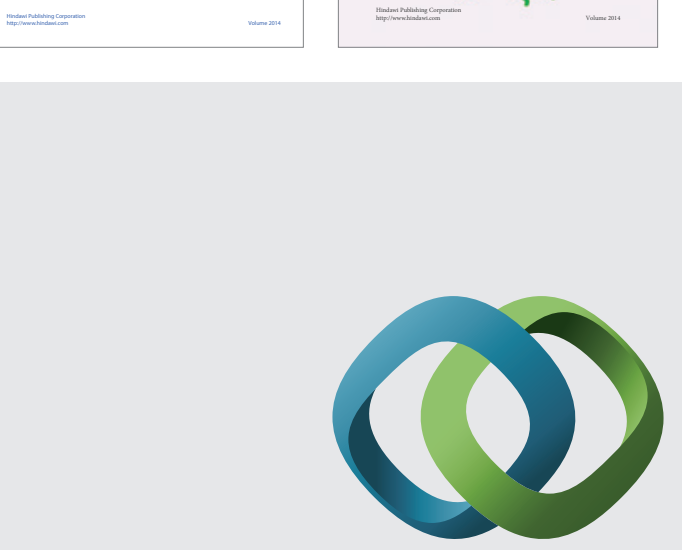

\section{Hindawi}

Submit your manuscripts at

http://www.hindawi.com
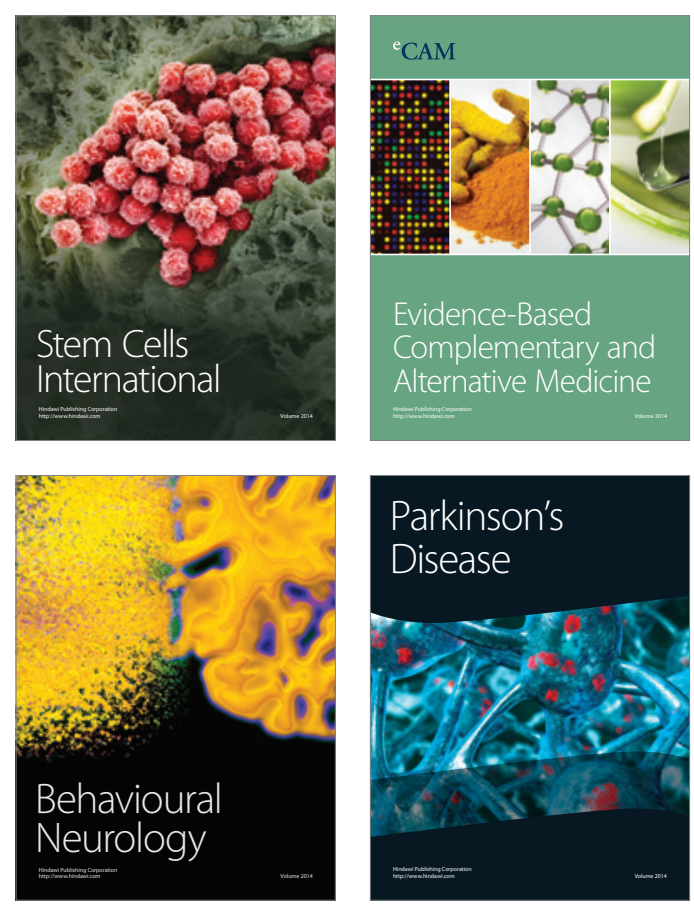

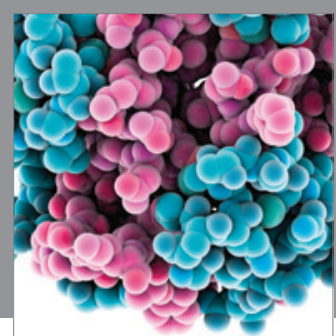

Journal of
Diabetes Research

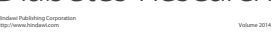

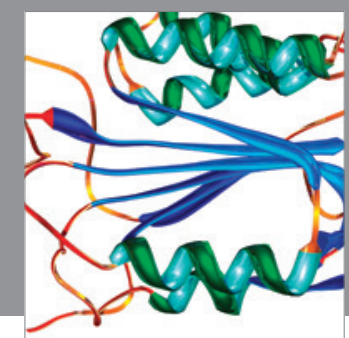

Disease Markers
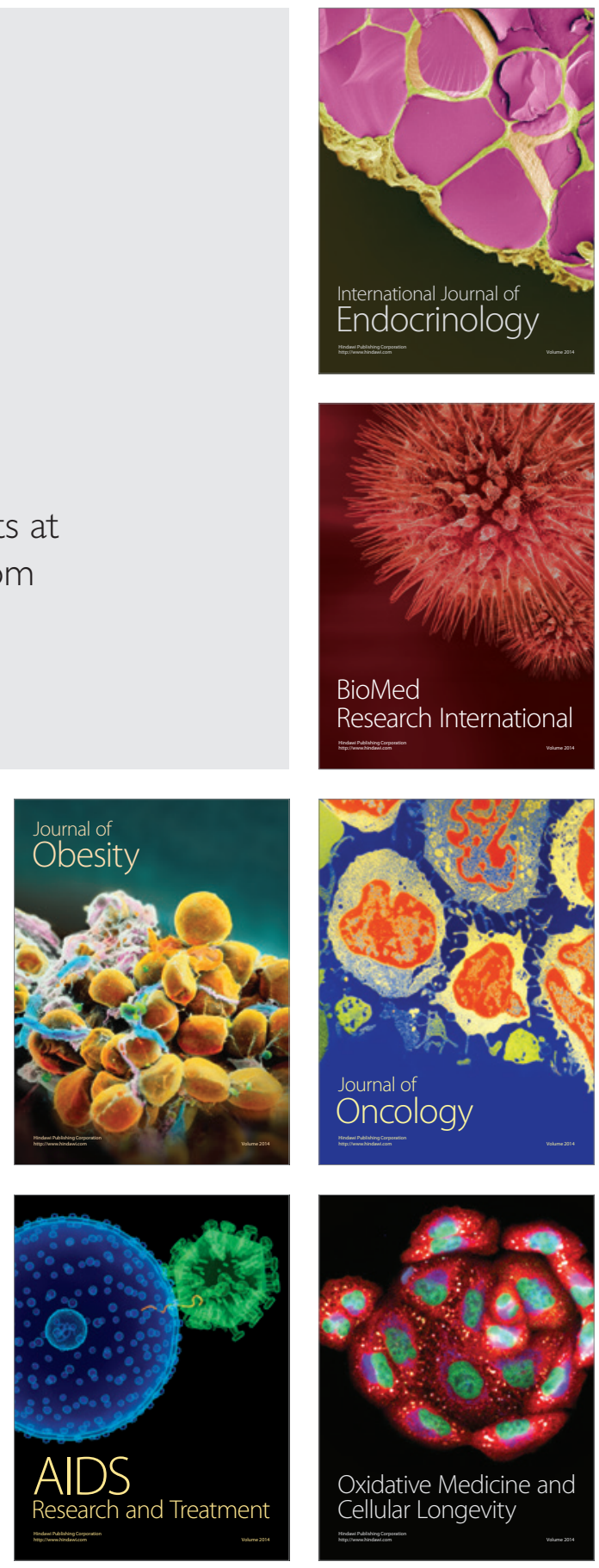